\title{
Systematic assessment of the tagging polymorphisms of the COL1A1 gene for high myopia
}

\author{
Chung-Ling Liang $\cdot$ Kuo-Sheng Hung • \\ Yueh-Ying Tsai $\cdot$ Wansu Chang $\cdot$ Hsin-Shih Wang · \\ Suh-Hang Hank Juo
}

Received: 22 November 2006/ Accepted: 8 January 2007 / Published online: 2 February 2007

(C) The Japan Society of Human Genetics and Springer 2007

\begin{abstract}
Reduced scleral collagen accumulation has been found in the development of myopia. Single nucleotide polymorphisms (SNPs) at the type I collagen $\alpha-1$ gene (COL1A1) may cause different susceptibilities to myopia. We conducted a case-control study to systematically examine COL1A1 as a candidate gene for high myopia. A case was defined as spherical refraction $\leq-6 \mathrm{D}$ and control $\geq-1.5 \mathrm{D}$. The study comprised 471 cases and 623 controls, and ten tagging
\end{abstract}

Electronic supplementary material The online version of this article (doi:10.1007/s10038-007-0117-6) contains supplementary material, which is available to authorized users.

\section{C.-L. Liang}

Bright-Eyes Clinic, Kaohsiung, Taiwan

C.-L. Liang · H.-S. Wang

Graduate Institute of Clinical Medical Sciences,

Chang Gung University, Taoyuan, Taiwan

K.-S. Hung

Clinical Research Center,

Taipei Medical University Wan Fang Hospital,

Taipei, Taiwan

Y.-Y. Tsai · W. Chang · S.-H. H. Juo ( $₫)$

Graduate Institute of Medical Genetics,

Kaohsiung Medical University,

100 TzYou First Road, Kaohsiung 807, Taiwan

e-mail:shj34@columbia.edu

S.-H. H. Juo

Department of Medical Research,

Kaohsiung Medical University Hospital,

Kaohsiung, Taiwan

S.-H. H. Juo

Department of Medical Research,

Mackay Memorial Hospital, Taipei, Taiwan
SNPs were genotyped. None of the SNPs reached the significant level of 0.05 . Subset analysis on cases with a strong family history did not demonstrate significant results. We could not find an interaction between gene and near work. Exploratory analyses by changing the cutoff values to re-define cases and controls did not improve the results. Haplotype analysis did not yield significant association with myopia. This study failed to demonstrate COL1A1 as a significant risk factor for high myopia.

Keywords SNP · COL1A1 - Haplotype - Myopia · Case-control study

\section{Introduction}

Myopia is a common eye condition worldwide. Taiwan is among the highest risk areas in the world. The prevalence of high myopia is $18 \%$ among young Taiwanese men and $24 \%$ among young Taiwanese women (Lin et al. 2001). Using family data, we reported that a family history was a significant risk factor for high myopia (Liang et al. 2004). However, genetic studies on non-syndromic myopia are relatively sparse. More efforts are needed to discover genetic loci influencing susceptibility to common myopia and to fill the gap in our knowledge of the underlying genetic mechanism.

The development of high myopia is associated with marked thinning of the sclera. Collagen accounts for $90 \%$ of scleral dry weight (Norton and Rada 1995), and the majority is type I collagen (Keeley et al. 1984). It was found in animal studies that type I collagen mRNA expression significantly decreased in the sclera of the myopic eyes relative to the contralateral control 
eyes (Gentle et al. 2003). The study also found that posterior scleral collagen degradation was significantly increased in eyes developing myopia. Genetic variants at the type I collagen $\alpha-1$ gene (COL1A1) may influence gene expression, which causes different susceptibilities to myopia. Paluru et al. (2003) reported a linkage of high myopia to $17 \mathrm{q} 21-22$, where COL1A1 is located. Accordingly, COL1A1 can be a candidate gene for myopia. Although Paluru et al. (2003) did not find causal mutations in the coding region of COL1A1, this does not completely exclude a possibility of a polymorphism in the non-coding region conferring a risk for myopia. We conducted a case-control study to test for the COL1A1 gene as a susceptibility locus to high myopia.

\section{Methods}

\section{Subjects}

The participants were recruited from young male military conscripts between 2003 and 2006. All subjects were of Han Chinese descent and between the ages of $18-25$ years. Individuals with spherical refraction $\leq-$ $6.0 \mathrm{D}$ in one eye and $\leq-4.0 \mathrm{D}$ in the other eye were classified as cases. A subject was recruited as a control if his worse eye had spherical refraction $\geq-1.5 \mathrm{D}$. The refractive error was measured with an autorefractometer (Topcon KR-8100) for all eyes. Each participant was given a questionnaire in which details of family history and environmental factors were asked. The details of the questionnaire can be found in our previous publication (Liang et al. 2004). All participants gave their informed consent. The study was approved by the Institutional Review Board at the Kaohsiung Medical University, Taiwan.

\section{SNP selection and genotyping}

Tagging single nucleotide polymorphisms (tSNPs) at COL1A1 were selected from the phase II data of the HapMap Project (Altshuler et al. 2005) using the Tagger Pairwise method (Barrett et al. 2005). Seven tSNPs were selected according to the following criteria: $r^{2} \geq 0.8$ and minor allele frequency (MAF) $\geq 10 \%$ in the Han Chinese population (http://www.hapmap.org). To increase the coverage of this candidate gene, we also manually selected three additional common SNPs (rs2075559, rs2249492 and rs1061237) with a MAF $>40 \%$. Genotyping was carried out by using the TaqMan technology. To reduce the genotyping cost, we did not type every subject for all SNPs. However, the number of genotyping for every SNP was more than 960 to assure enough statistical power.

\section{Statistics}

Hardy-Weinberg equilibrium (HWE) was tested among cases and controls separately. We examined the effect of the common allele in three genetic models (dominant, additive and recessive). We also used the Hap-Clustering program (Tzeng et al. 2006) to perform haplotype analysis for each haplotype block. Education level is a surrogate of near work, and it has been repeatedly shown as a risk factor for myopia (Mutti et al. 2002; Liang et al. 2004). We further tested whether education can be an effect modifier to interact with the genotypes. The education level was dichotomized into high (i.e., college or graduate) education level and low education level, to be consistent with our previous study (Liang et al. 2004). To enrich the genetic component, we also performed a subset analysis only on cases with highly myopic parents.

We used 450 cases and 550 controls to estimate the power to detect two different odds ratios given the risk allele frequency of $10 \%$ in the general population. The results showed that our data provided a power of $93 \%$ and $79 \%$ for an odds ratio (OR) of 1.9 and 1.7, respectively.

\section{Results}

A total of 471 cases and 623 controls was included. SNP rs2141279 was not in HWE $\left(P<1.0 \times 10^{-9}\right)$ and was removed for further statistical analysis. None of the SNPs reached a nominal significant level of 0.05 (Table 1). Subset analysis on cases with highly myopic parents did not yield any new significant result except for rs2277633, with a $P$ value of 0.04 under the recessive model (supplementary Table 1). Similarly, subjects stratified by educational level did not demonstrate any significant result or a significant gene $\times$ education interaction (supplementary Table 2). We further performed exploratory analyses by increasing the cutoff values to re-define cases $(\leq-7 \mathrm{D},-8 \mathrm{D},-9 \mathrm{D}$ or $-10 \mathrm{D})$ and decreasing the cutoff values to re-define controls $(\geq-1.0 \mathrm{D}$ or $\geq-0.5 \mathrm{D})$. However, the re-defined cases and controls did not improve the results. The ten SNPs form three small haplotype blocks, but haplotype analyses did not yield significant results for any block (overall $P>0.9$ for each block). 
Table 1 Ten SNPs and their relationships with high myopia under three genetic models (dominant, additive and recessive)

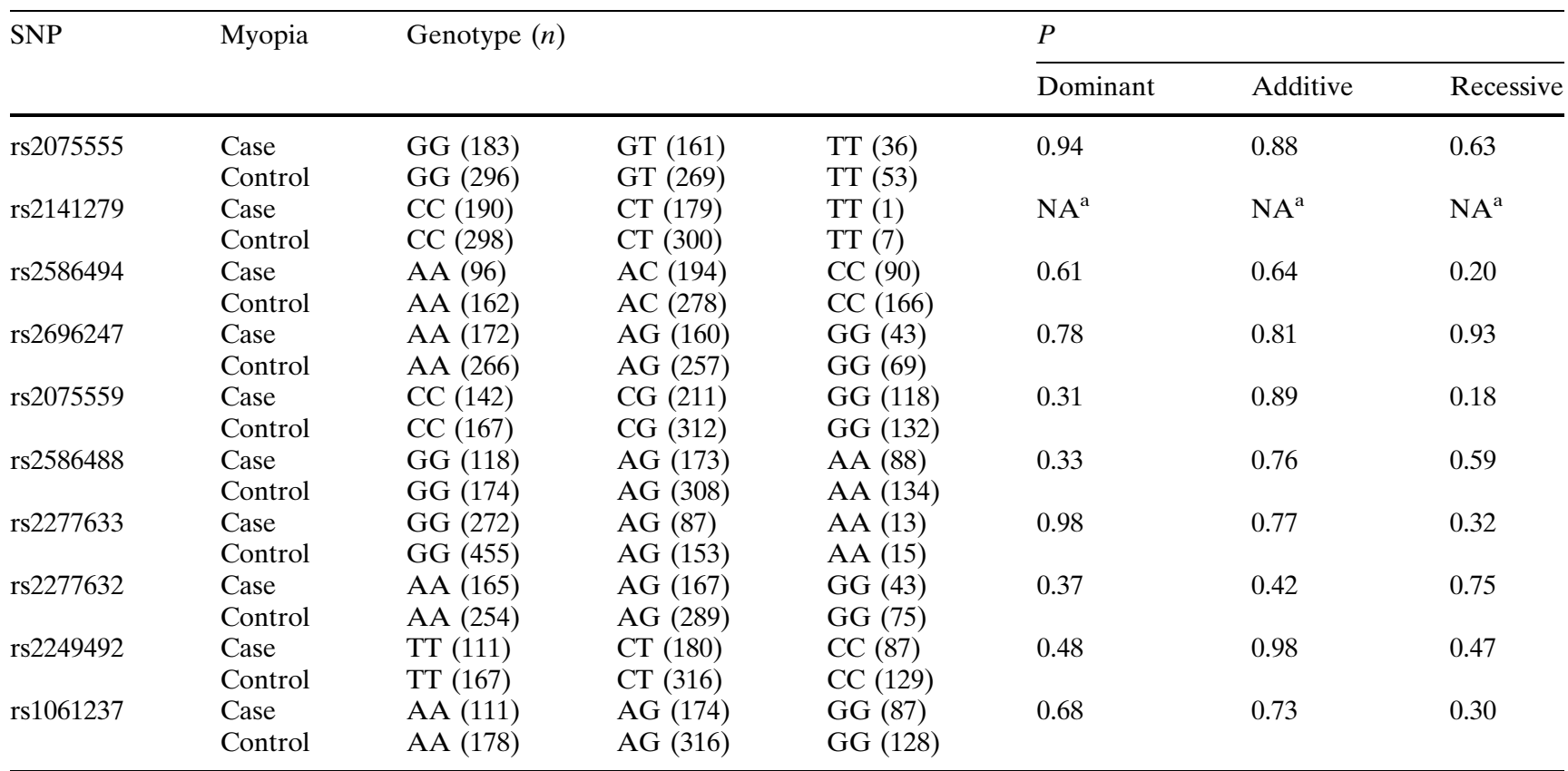

${ }^{a}$ No statistical results because not in HWE

\section{Discussion}

We systematically investigated ten tSNPs at COL1A1 using a large Taiwanese population. We analyzed the genetic effects of each SNP on high myopia, but none of them was significantly associated with the phenotype. Various analyses were also performed, including: subset analysis on subjects with a strong family history; subset analysis according to education level; and analyses based on different cutoff points to re-define cases and controls. Although we found one $P$ value of 0.04 for the cases with a strong family history, this $P$ value should not be considered significant after adjustment for multiple testing. No other approaches yielded any significant finding. Given the large sample size in the present study, and the use of tSNPs, it is unlikely that the existence of a common genetic variant at COL1A1 would influence susceptibility to high myopia.

Our study design has strengths and limitations. We recruited only young men to avoid the heterogeneity due to gender in the phenotype, because women have been shown to have a higher risk for high myopia (Liang et al. 2004). Therefore, our study was not confounded by the gender issue. The study population was relatively homogenous in terms of ethnicity, geographic location and age. As a result, several undetectable confounding factors could be minimized. It needs to be noted that our results cannot exclude the existence of a rare causal genetic polymorphism at COLIA1. Using both empirical and simulated data,
Zeggini et al. (2005) showed that common tSNPs have limited capacity to tag variants with minor allele frequencies of $<5 \%$. Although using haplotypes can increase the power to detect a rare causal allele, the power is still not satisfied (de Bakker et al. 2005).

We did not examine the Sp1 polymorphism that is the most commonly studied marker at COLIA1, because it is not polymorphic in East Asians (Lei et al. 2003).

In conclusion, our systematic evaluation indicated that the COL1A1 gene is unlikely to play an important role in high myopia.

Acknowledgments This work was supported by grants from the National Research Program of Genomic Medicine, Taiwan, NSC95-3112-B-037 (C.L.L. and S.H.J.), and the intramural funding at the Kaohsiung Medical University KMU-QC-094001 (W.C. and S.H.J.).

\section{References}

Altshuler D, Brooks LD, Chakravarti A, Collins FS, Daly MJ, Donnelly P, International HapMap Consortium (2005) A haplotype map of the human genome. Nature 437:1299-1320

Barrett JC, Fry B, Maller J, Daly MJ (2005) Haploview: analysis and visualization of LD and haplotype maps. Bioinformatics 21:263-265

de Bakker PI, Yelensky R, Pe'er I, Gabriel SB, Daly MJ, Altshuler D (2005) Efficiency and power in genetic association studies. Nat Genet 37:1217-1223

Gentle A, Liu Y, Martin JE, Conti GL, McBrien NA (2003) Collagen gene expression and the altered accumulation of 
scleral collagen during the development of high myopia. J Biol Chem 278:16587-16594

Keeley FW, Morin JD, Vesely S (1984) Characterization of collagen from normal human sclera. Exp Eye Res 39:533542

Liang CL, Yen E, Su JY, Liu C, Chang TY, Park N, Wu MJ, Lee S, Flynn JT, Juo SH (2004) The impact of the family history of high myopia on level and onset of myopia. Invest Ophthalmol Vis Sci 45:3446-3452

Lin LL, Shih YF, Hsiao CK, Chen CJ, Lee LA, Hung PT (2001) Epidemiologic study of the prevalence and severity of myopia among schoolchildren in Taiwan in 2000. J Formos Med Assoc 100:684-691

Mutti DO, Mitchell GL, Moeschberger ML, Jones LA, Zadnik K (2002) Parental myopia, near work, school achievement, and children's refractive error. Invest Ophthalmol Vis Sci 43:3633-3640

Norton TT, Rada JA (1995) Reduced extracellular matrix in mammalian sclera with induced myopia. Vision Res 35:1271-1281
Paluru P, Ronan SM, Heon E, Devoto M, Wildenberg SC, Scavello G, Holleschau A, Makitie O, Cole WG, King RA, Young TL (2003) New locus for autosomal dominant high myopia maps to the long arm of chromosome 17. Invest Ophthalmol Vis Sci 44:1830-1836

Tzeng JY, Wang CH, Kao JT, Hsiao CK (2006) Regressionbased association analysis with clustered haplotypes through use of genotypes. Am J Hum Genet 78:231-242

Zeggini E, Rayner W, Morris AP, Hattersley AT, Walker M, Hitman GA, Deloukas P, Cardon LR, McCarthy MI (2005) An evaluation of HapMap sample size and tagging SNP performance in large-scale empirical and simulated data sets. Nat Genet 37:1320-1322

Lei SF, Deng FY, Liu XH, Huang QR, Qin Y, Zhou Q, Jiang DK, Li YM, Mo XY, Liu MY, Chen XD, Wu XS, Shen H, Dvornyk V, Zhao L, Recker RR, Deng HW (2003) Polymorphisms of four bone mineral density candidate genes in Chinese populations and comparison with other populations of different ethnicity. J Bone Miner Metab 21:34-42 